\title{
Recent advances and impacts of microtiter plate-based fermentations in synthetic biology and bioprocess development
}

\begin{abstract}
Microscale fermentation has totally revolutionized the way microbial cell cultures are studied and developed, especially in the fields of industrial microbiology, synthetic biology, and bioprocess design among others, where fermentation usually needs to be optimized and higher throughput is thus beneficial. Synthetic biology is a multidisciplinary field focusing on developing foundational principles and technologies to ultimately enable a systematic forward-engineering of biological parts and systems for improved and novel applications. The easy automation of these systems is also an important advantage. They allow the continuous, high-throughput, parallel measurement of kinetic growth data and have the potential to be easily integrated into automated platforms for further sample processing. An important aspect of the lab-bench scale bioreactor is the fed-batch operation capacity, which allows microbial substrate-limited growth to be performed and minimizes carbon overflow while greatly improving production yields. Automation not only allows the sample throughput to be increased, but also increases fermentation capabilities and complexity.
\end{abstract}

